\title{
Lyapunov stability analysis of higher-order 2-D systems
}

\author{
Chiaki Kojima
}

\author{
Paolo Rapisarda
}

\author{
Kiyotsugu Takaba
}

\begin{abstract}
We give necessary and sufficient conditions, based on the existence of a Lyapunov functional, for the asymptotic stability of a square autonomous 2-D behavior in the sense of Valcher. We also show how Lyapunov functionals can be computed solving a two-variable polynomial equation, and we give necessary and sufficient conditions for the solvability of this equation for scalar systems.
\end{abstract}

\section{INTRODUCTION}

Discrete- and continuous-time two-dimensional (in the following abbreviated as 2-D) systems have application in all those situations when the evolution of the to-be-modeled system depends on two independent variables, for example time and (one-dimensional) space as it happens when analyzing the vibrations of structures, or in iterative learning control; or two spatial variables, for example in the case of digital image processing, in physics, etc. These systems have a number of applications, for example in seismology, image enhancement, circuit theory, and so forth.

The notion of stability, because of its important consequences in the analysis and design of control systems and of filters, has attracted considerable interest also in the case of 2-D systems. The issue of what a reasonable definition of stability is for this situation presents first and foremost the difficulty of extending the notion of "past" and "future", so self-evident in the 1-D framework, to the case of two independent variables, where there is no obvious such splitting. An eminently reasonable position is to let the laws describing the physical phenomenon themselves dictate what the direction is of the evolution of the system. This is the approach pioneered by M.E. Valcher in [17] and followed in this paper. Central in this framework are the notions of "characteristic set" and "characteristic cone" of a behavior, which we will introduce in the next section; a linear, shift-invariant behavior which admits a nontrivial (or "proper") characteristic cone is called stable if its trajectories go asymptotically to zero within the "future cone". In [17] algebraic tests are given which, starting from the description of a system as the kernel of a polynomial operator in the shifts, determine whether a cone is characteristic for the system or not (see Proposition 2.9 of [17]); and whether a

Department of Information Physics and Computing, Graduate School of Information Science and Technology, The University of Tokyo, Hongo, Bunkyo-Ku, Tokyo 113-0033, Japan tel/fax: +81-3-5841-6890; e-mail: chiaki_kojima@ipc.i.u-tokyo.ac.jp

Information: Signals, Images, Systems group, School of Electronics and Computer Science, University of Southampton, SO171BJ Southampton, United Kingdom, fax: +44- (023)- 80594498; e-mail: pr3 decs.soton.ac.uk

Dept. of Applied Mathematics and Physics, Graduate Schoo of Informatics, Kyoto University, Kyoto 606-8501, Japan, email: takabaeamp.i.kyoto-u.ac.jp system is stable or not. These tests are based on the location of the points of an algebraic variety associated with the polynomial matrix inducing a kernel representation of the system.

In this paper we present a necessary and sufficient condition for the asymptotic stability of 2-D systems based on Lyapunov functions. This idea is by no means original, having been applied already in [11], [1]; however, those approaches relied entirely on a specific ("state-space") type of representation of the system, while we deal with systems described in a general form, namely as the solutions of a system of partial difference equations. We adopt the behavioral framework pioneered by J.C. Willems in the 1-D case (see [15]), and extended to the 2-D case by P. Rocha (see [16]) and other authors (see [13], [14], [17]). In this setting the main object of study is the behavior, the set consisting of all the trajectories admissible by the physical laws describing the system trajectories.

In this paper, the concepts and tools of the behavioral approach, and of quadratic differential forms will be put to strenuous use. The reader not familiar with them is referred to [14], [15], [18] for a thorough exposition. We include some background material on 2-D behaviors and quadratic differential forms in sections II and section III. Section IV contains the main result of this paper, namely a stability criterion for higher-order systems of differential- or difference equations based on Lyapunov analysis. Section V discusses the current research directions being pursued.

Notation: We denote with $\mathbb{R}^{\mathrm{r} \times \mathrm{w}}\left[\xi_{1}, \xi_{2}\right]$ (respectively, $\left.\mathbb{R}^{\mathrm{r} \times \mathrm{w}}\left[\xi_{1}, \xi_{2}, \xi_{1}^{-1}, \xi_{2}^{-1}\right]\right)$ the set of all $r \times \mathrm{w}$ matrices with entries in the ring $\mathbb{R}\left[\xi_{1}, \xi_{2}\right]$ of polynomials in 2 indeterminates, with real coefficients (respectively in the ring $\mathbb{R}\left[\xi_{1}, \xi_{2}, \xi_{1}^{-1}, \xi_{2}^{-1}\right]$ of Laurent polynomials in 2 indeterminates with real coefficients). Given a nonzero Laurent polynomial $p\left(\xi_{1}, \xi_{2}\right)=\sum_{\ell, m} p_{\ell, m} \xi_{1}^{\ell} \xi_{2}^{m} \in \mathbb{R}\left[\xi_{1}, \xi_{2}, \xi_{1}^{-1}, \xi_{2}^{-1}\right]$, the Laurent variety of $p$ is defined as

$$
\mathcal{V}_{L}(p):=\{(\alpha, \beta) \in \mathbb{C} \times \mathbb{C} \mid \alpha \beta \neq 0, p(\alpha, \beta)=0\}
$$

This definition extends to sets $\mathcal{I}$ of Laurent polynomials, with $\mathcal{V}(\mathcal{I})$ being the intersection of the Laurent varieties of all polynomials in the set. For $R \in \mathbb{R}^{\mathrm{r} \times \mathrm{w}}\left[\xi_{1}, \xi_{2}, \xi_{1}^{-1}, \xi_{2}^{-1}\right]$, the characteristic ideal is the ideal of $\mathbb{R}\left[\xi_{1}, \xi_{2}\right]$ generated by the determinants of all $\mathrm{w} \times \mathrm{w}$ minors of $R$, and the characteristic variety is the set of roots common to all polynomials in the ideal. Further properties and definitions can be found in [3].

A set $\mathcal{K} \subset \mathbb{R} \times \mathbb{R}$ is called a cone if $\alpha \mathcal{K} \subset \mathcal{K}$ for all $\alpha \geq 0$. A cone is solid if it contains an open ball in $\mathbb{R} \times \mathbb{R}$, and pointed if $\mathcal{K} \cap-\mathcal{K}=\{(0,0)\}$. A cone is proper if it is closed, 
pointed, solid, and convex. It is easy to see that a proper cone is uniquely identified as the set of nonnegative linear combinations of two linearly independent vectors $v_{1}, v_{2} \in$ $\mathbb{R}^{2}$. In the following we will often consider the intersection of a cone $\mathcal{K}$ with $\mathbb{Z} \times \mathbb{Z}$; whenever it will be clear from the context, we will be denoting this set with $\mathcal{K}$ instead of with $\mathcal{K} \cap \mathbb{Z} \times \mathbb{Z}$.

We denote with $\overline{\mathcal{P}_{1}}$ the closed unit polydisk:

$$
\overline{\mathcal{P}_{1}}:=\{(\alpha, \beta) \in \mathbb{C} \times \mathbb{C}|| \alpha|\leq 1,| \beta \mid \leq 1\}
$$

Given a set $\mathcal{S} \subset \mathbb{Z} \times \mathbb{Z}$, its (discrete) convex hull is the intersection of the convex hull of $\mathcal{S}$ (seen as a subset of $\mathbb{R} \times \mathbb{R}$ ) and of $\mathbb{Z} \times \mathbb{Z}$. In the following we will also refer to the (discrete) convex hull associated with an element $p \in$ $\mathbb{R}\left[\xi_{1}, \xi_{2}, \xi_{1}^{-1}, \xi_{2}^{-1}\right]$, meaning the (discrete) convex hull of the support of $p$, i.e. the set

$$
\begin{aligned}
\operatorname{supp}(p):=\{ & \left(x_{1}, x_{2}\right) \in \mathbb{Z} \times \mathbb{Z} \mid \text { the coefficient of } \xi_{1}^{h} \xi_{2}^{k} \\
& \text { in } \left.p\left(\xi_{1}, \xi_{2}, \xi_{1}^{-1}, \xi_{2}^{-1}\right) \text { is } \neq 0\right\}
\end{aligned}
$$

We denote with $\mathbb{W}^{\mathbb{T}}$ the set consisting of all trajectories from $\mathbb{T}$ to $\mathbb{W}$. We denote with $\sigma_{1}, \sigma_{2}$ the shift operators defined as

$$
\begin{aligned}
\sigma_{i}:\left(\mathbb{R}^{\mathrm{w}}\right)^{\mathbb{Z}^{2}} \rightarrow\left(\mathbb{R}^{\mathrm{w}}\right)^{\mathbb{Z}^{2}} i=1,2 \\
\left(\sigma_{1} w\right)\left(x_{1}, x_{2}\right):=w\left(x_{1}-1, x_{2}\right) \\
\left(\sigma_{2} w\right)\left(x_{1}, x_{2}\right):=w\left(x_{1}, x_{2}-1\right)
\end{aligned}
$$

\section{2-D BEHAVIORS}

In behavioral system theory, the behavior is a subset of the set $\mathbb{W}^{\mathbb{T}}$. In this paper we consider systems with $\mathbb{T}=\mathbb{Z}^{2}$ (from which the terminology "2-D-system" derives) and $\mathbb{W}=\mathbb{R}^{\mathrm{w}}$. We call $\mathfrak{B}$ a linear discrete-time complete 2-D behavior if it is the solution set of a system of linear, constant-coefficient difference equations with two independent variables; more precisely, if $\mathfrak{B}$ is the subset of $\left(\mathbb{R}^{\mathrm{w}}\right)^{\mathbb{Z}^{2}}$ consisting of all solutions to

$$
R\left(\sigma_{1}, \sigma_{2}\right) w=0
$$

where $R \in \mathbb{R}^{\mathrm{r} \times \mathrm{w}}\left[\xi_{1}, \xi_{2}, \xi_{1}^{-1}, \xi_{2}^{-1}\right]$. We call (1) a kernel representation of $\mathfrak{B}$. We denote the set consisting of all linear discrete-time complete 2-D behaviors with w external variables with $\mathcal{L}_{2}^{\mathrm{W}}$.

$\mathfrak{B} \in \mathcal{L}_{2}^{\mathrm{W}}$ is autonomous if there exists a proper cone $\mathcal{K} \subset$ $\mathbb{R} \times \mathbb{R}$ such that

$$
\left[w_{1}, w_{2} \in \mathfrak{B} \text { and } w_{1 \mid \mathcal{K} \cap \mathbb{Z} \times \mathbb{Z}}=w_{2 \mid \mathcal{K} \cap \mathbb{Z} \times \mathbb{Z}}\right] \Longrightarrow\left[w_{1}=w_{2}\right]
$$

Such a cone $\mathcal{K} \cap \mathbb{Z} \times \mathbb{Z}$ will be called a proper characteristic cone for $\mathfrak{B}$. Observe that if $w \in \mathfrak{B}$ is such that $w_{\mid \mathcal{K} \cap \mathbb{Z} \times \mathbb{Z}}=0$, then $w=0$.

Proper characteristic cones play an important role in the definition of stability of a 2-D system according to Valcher, and we now proceed to characterize them algebraically, following closely the treatment of [17]. The following result holds.

Theorem 1: Let $\mathfrak{B} \in \mathcal{L}_{2}^{\mathrm{w}}$ be autonomous, and let $\mathfrak{B}=$ ker $R\left(\sigma_{1}, \sigma_{2}\right)$ for some $R \in \mathbb{R}^{\mathrm{r} \times \mathrm{w}}\left[\xi_{1}, \xi_{2}, \xi_{1}^{-1}, \xi_{2}^{-1}\right]$. Then there exist $H \in \mathbb{R}^{\bullet} \times\left[\xi_{1}, \xi_{2}, \xi_{1}^{-1}, \xi_{2}^{-1}\right]$ right factor prime, and $\Delta \in \mathbb{R}^{\mathrm{w} \times \mathrm{w}}\left[\xi_{1}, \xi_{2}, \xi_{1}^{-1}, \xi_{2}^{-1}\right]$ nonsingular, such that $R=$ $H \cdot \Delta$.

Moreover, denote $\delta:=\operatorname{det}(\Delta) \in \mathbb{R}\left[\xi_{1}, \xi_{2}, \xi_{1}^{-1}, \xi_{2}^{-1}\right]$. The following statements are equivalent:

1) The proper cone $\mathcal{K}$ is characteristic for $\mathfrak{B}$;

2) The proper cone $\mathcal{K}$ is characteristic for ker $\Delta\left(\sigma_{1}, \sigma_{2}\right)$;

3) The proper cone $\mathcal{K}$ is characteristic for $\operatorname{ker} \delta\left(\sigma_{1}, \sigma_{2}\right)$;

4) The discrete convex hull $\mathcal{H}_{\delta}$ of $\delta$ satisfies the following two conditions:

$$
\begin{array}{ll}
\text { 4a. } & -\mathcal{H}_{\delta} \subset \mathcal{K} ; \\
\text { 4.b. } & -\mathcal{H}_{\delta} \subset \mathcal{K} \text { intersects the generating lines of } \\
& \mathcal{K} \text { only in }(0,0) .
\end{array}
$$

It can be shown (see [2]) that if $\mathfrak{B} \in \mathcal{L}_{2}^{\text {W }}$ is such that $\mathfrak{B}=\operatorname{ker} R\left(\sigma_{1}, \sigma_{2}\right)$ for some right factor prime matrix $R \in \mathbb{R}^{\mathrm{r} \times \mathrm{w}}\left[\xi_{1}, \xi_{2}, \xi_{1}^{-1}, \xi_{2}^{-1}\right]$, then $\mathfrak{B}$ is autonomous and finitedimensional. It can be shown (see Lemma 2.4 of [17]) that in this case, every proper cone is characteristic for $\mathfrak{B}$.

If $\mathfrak{B}$ is autonomous, and $\mathfrak{B}=\operatorname{ker} R\left(\sigma_{1}, \sigma_{2}\right)$ for some nonsingular Laurent matrix $R$, then $\mathfrak{B}$ is called a square autonomous behavior. Observe that Theorem 1 shows that for any autonomous behavior $\mathfrak{B}$ whose kernel representation can be factored as $H \Delta$ with $H$ right factor prime and $\Delta$ nonsingular, the characteristic cone is determined by its "square autonomous part" ker $\Delta\left(\sigma_{1}, \sigma_{2}\right)$.

We now discuss in some detail the concept of stability introduced by Valcher in [17]. In order to do so, we need to distinguish the finite-dimensional and the square autonomous case. In the former case, where each $w \in \mathfrak{B}$ is uniquely determined by its values in a finite subset of $\mathbb{Z} \times \mathbb{Z}$, the definition is as follows.

Definition 2: Let $\mathfrak{B} \in \mathcal{L}_{2}^{\mathrm{W}}$ be autonomous and finitedimensional, and let $\mathcal{K}$ be any proper cone of $\mathbb{Z} \times \mathbb{Z}$. $\mathfrak{B}$ is $\mathcal{K}$-stable if

$$
[w \in \mathfrak{B}] \Longrightarrow\left[\lim _{\substack{(i, j) \in \mathcal{K} \\|i|+|j| \rightarrow+\infty}}\|w(i, j)\|=0\right]
$$

The following algebraic characterization of finitedimensional stable behaviors (see Theorem 3.3 p. 297 of [17]) holds. In order to avoid cumbersome details, we follow [17], and only consider proper cones generated by unimodular integer matrices, which are then isomorphic to the first orthant of $\mathbb{Z} \times \mathbb{Z}$, in the sense that there exists a (linear, bijective) transformation $T: \mathbb{Z}^{2} \rightarrow Z^{2}$ such that $T(\mathcal{K})$ is the first orthant.

Theorem 3: Let $\mathfrak{B}=\operatorname{ker} H\left(\sigma_{1}, \sigma_{2}\right)$, with

$$
H\left(\xi_{1}, \xi_{2}\right)=\sum_{\ell, m} H_{\ell, m} \xi_{1}^{\ell} \xi_{2}^{m} \in \mathbb{R}^{\bullet \times \mathrm{w}}\left[\xi_{1}, \xi_{2}, \xi_{1}^{-1}, \xi_{2}^{-1}\right]
$$

right factor prime, and let $\mathcal{K}$ be a proper cone isomorphic to the first orthant. Denote the transformation mapping $\mathcal{K}$ to the first orthant with $T$, and with $\left(t_{1}(\ell, m), t_{2}(\ell, m)\right)$ the image of $(\ell, m) \in \mathbb{Z} \times \mathbb{Z}$ under $T$. Define

$$
H_{T}\left(\xi_{1}, \xi_{2}\right):=\sum_{\ell, m} H_{\ell, m} \xi_{1}^{t_{2}(\ell, m)} \xi_{2}^{t_{1}(\ell, m)}
$$

Then the following two statements are equivalent:

1) $\mathfrak{B}$ is $\mathcal{K}$-stable; 
2) Every $(\alpha, \beta)$ in the Laurent variety of the maximal order minors of $H_{T}$ satisfies $|\alpha|>1$ and $|\beta|>1$.

The definition of stability in the square autonomous case takes into account the fact that since the set of points in which a trajectory can be freely assigned is infinite, it may happen that particular choices of the "initial conditions" correspond to trajectories of the behavior which do not die out within a proper cone $\mathcal{K}$. In order to state the definition of stability for the square case, we need to introduce the following notation: given a proper cone $\mathcal{C}$, we denote with $\delta(\mathcal{C})$ the boundary of $\mathcal{C}$, i.e. the generating lines of $\mathcal{C}$. Moreover, we denote with $(\delta(\mathcal{C}))^{n}$ the set consisting of the points of $\mathbb{Z} \times \mathbb{Z}$ whose distance from $\delta(\mathcal{C})$ is less than $n$, i.e.

$$
\begin{aligned}
(\delta(\mathcal{C}))^{n}:= & \{(i, j) \in \mathbb{Z} \times \mathbb{Z} \mid \min \{|i-h|+|j-k| \\
& \text { with } \left.\left.\left(x_{1}, x_{2}\right) \in \delta(\mathcal{C})\right\} \leq n\right\}
\end{aligned}
$$

The definition of $\mathcal{K}$-stable square autonomous behavior is as follows.

Definition 4: Let $\mathcal{K}$ be a proper cone such that $-\mathcal{K}$ is characteristic for a square autonomous behavior $\mathfrak{B} \in \mathcal{L}_{2}^{\mathrm{W}} \cdot \mathfrak{B}$ is $\mathcal{K}$-stable if there exists some positive integer $n$ such that

$$
\begin{aligned}
& {\left[w \in \mathfrak{B}, w \text { bounded in }(\delta(-\mathcal{K}))^{n}\right]} \\
& \Longrightarrow\left[\begin{array}{l}
\left.\lim _{\substack{i, j \\
|i|}}\|w(i, j)\|=0\right] \\
|i| j \mid \rightarrow+\infty
\end{array}\right.
\end{aligned}
$$

The following is an algebraic characterization of $\mathcal{K}$-stability (see Theorem 3.6 of [17] for a proof).

Theorem 5: Let $\mathfrak{B}=$ ker $\Delta\left(\sigma_{1}, \sigma_{2}\right)$ be a square autonomous behavior, and let $\mathcal{K}$ be a proper cone which is $T$-isomorphic to the first orthant. Denote $\delta:=\operatorname{det}(\Delta)$, and assume w.l.o.g. that $\mathcal{H}_{\delta} \subset \mathcal{K}$ and that $\mathcal{H}_{\delta} \cap \delta \mathcal{K}=\{(0,0)\}$. Denote with $\left(t_{1}(\ell, m), t_{2}(\ell, m)\right)$ the image of $(\ell, m) \in \mathbb{Z} \times \mathbb{Z}$ under $T$. Define

$$
\Delta_{T}\left(\xi_{1}, \xi_{2}\right):=\sum_{\ell, m} \Delta_{\ell, m} \xi_{1}^{t_{1}(\ell, m)} \xi_{2}^{t_{2}(\ell, m)}
$$

Then the following two statements are equivalent:

1) $\mathfrak{B}$ is $\mathcal{K}$-stable;

2) The Laurent variety of det $\Delta_{T}$ does not intersect the closed unit polydisk $\overline{\mathcal{P}_{1}}$.

In section IV we will establish an equivalent characterization of $\mathcal{K}$ stability for the square autonomous case, which will be useful for the purposes of computing Lyapunov functions for a given behavior. We proceed in the next section to review some important concepts related to quadratic difference forms.

\section{BILINEAR- AND QUADRATIC DIFFERENCE FORMS FOR 2-D SYSTEMS}

In many modeling and control problems for linear systems it is necessary to study bilinear- and quadratic functionals of the system variables and their shifts (or their derivatives in the continuous-time case). For finite-dimensional continuoustime linear systems, an efficient representation for such functionals by means of two-variable polynomial matrices was introduced in [18]; in order to represent bilinear- and quadratic functionals of the variables of continuous-time 2-D-systems, 4-variable polynomial matrices are used (see [14]).

In the 1-D discrete-time case, quadratic difference forms have been introduced in [7]. We now examine the extension of quadratic difference forms to the 2-D discrete setting; some preliminary results in this sense have been obtained in [10].

In order to simplify the notation, define the multi-indices $\mathbf{k}:=\left(k_{1}, k_{2}\right), \mathbf{l}:=\left(l_{1}, l_{2}\right)$, and the notation $\zeta:=\left(\zeta_{1}, \zeta_{2}\right)$ and $\eta:=\left(\eta_{1}, \eta_{2}\right)$, and define $\zeta^{\mathbf{k}} \eta^{1}:=\zeta_{1}^{k_{1}} \zeta_{2}^{k_{2}} \eta_{1}^{l_{1}} \eta_{2}^{l_{2}}$.

Let $\mathbb{R}^{\mathrm{w}_{1} \times \mathrm{w}_{2}}[\zeta, \eta]$ denote the set of real polynomial $\mathrm{w}_{1} \times \mathrm{w}_{2}$ matrices in the 4 indeterminates $\zeta_{i}$ and $\eta_{i}, i=1,2$; that is, an element of $\mathbb{R}^{\mathrm{w}_{1} \times \mathrm{w}_{2}}[\zeta, \eta]$ is of the form

$$
\Phi(\zeta, \eta)=\sum_{\mathbf{k}, 1} \Phi_{\mathbf{k}, 1} \zeta^{\mathbf{k}} \eta^{\mathbf{l}}
$$

where $\Phi_{\mathbf{k}, 1} \in \mathbb{R}^{\mathrm{w}_{1} \times \mathrm{w}_{2}}$; the sum ranges over the nonnegative multi-indices $\mathbf{k}$ and $\mathbf{l}$, and is assumed to be finite. Such matrix induces a bilinear difference form (BDF in the following) $L_{\Phi}$

$$
\begin{aligned}
& L_{\Phi}:\left(\mathbb{R}^{w_{1}}\right)^{\mathbb{Z}^{2}} \times\left(\mathbb{R}^{w_{2}}\right)^{\mathbb{Z}^{2}} \longrightarrow(\mathbb{R})^{\mathbb{Z}^{2}} \\
& L_{\Phi}(v, w):=\sum_{\mathbf{k}, 1}\left(\sigma^{\mathbf{k}} v\right)^{\top} \Phi_{\mathbf{k}, \mathbf{l}}\left(\sigma^{\mathbf{l}} w\right)
\end{aligned}
$$

where the $\mathbf{k}$-th shift operator $\sigma^{\mathbf{k}}$ is defined as $\sigma^{\mathbf{k}}:=\sigma_{1}^{k_{1}} \sigma_{2}^{k_{2}}$, and analogously for $\sigma^{1}$.

The 4 -variable polynomial matrix $\Phi\left(\zeta_{1}, \zeta_{2}, \eta_{1}, \eta_{2}\right)$ is called symmetric if $\mathrm{w}_{1}=\mathrm{w}_{2}=$ : $\mathrm{w}$ and $\Phi\left(\zeta_{1}, \zeta_{2}, \eta_{1}, \eta_{2}\right)=$ $\Phi\left(\eta_{1}, \eta_{2}, \zeta_{1}, \zeta_{2}\right)^{\top}$, concisely written as $\Phi(\zeta, \eta)=\Phi(\eta, \zeta)^{\top}$. In this case, $\Phi$ induces also a quadratic functional

$$
\begin{aligned}
& Q_{\Phi}:\left(\mathbb{R}^{w}\right)^{\mathbb{Z}^{2}} \longrightarrow(\mathbb{R})^{\mathbb{Z}^{2}} \\
& Q_{\Phi}(w):=L_{\Phi}(w, w)
\end{aligned}
$$

We will call $Q_{\Phi}$ the quadratic difference form (in the following abbreviated with QDF) associated with the fourvariable polynomial matrix $\Phi$.

In this paper we also consider vectors $\Psi \in$ $\left(\mathbb{R}^{\mathrm{w}_{1} \times \mathrm{w}_{2}}[\zeta, \eta]\right)^{2}$, i.e.

$$
\Psi(\zeta, \eta)=\left[\begin{array}{l}
\Psi_{1}(\zeta, \eta) \\
\Psi_{2}(\zeta, \eta)
\end{array}\right]=: \operatorname{col}\left(\Psi_{i}(\zeta, \eta)\right)_{i=1,2}
$$

with $\Psi_{i} \in \mathbb{R}^{\mathrm{w}_{1} \times \mathrm{w}_{2}}[\zeta, \eta]$ and with $\operatorname{col}\left(A_{i}\right)_{i=1,2}$ the matrix obtained by stacking the two matrices $A_{i}$, both with the same number of columns, on top of each other. Such $\Psi$ induces a vector bilinear difference form (abbreviated $V B D F$ ), defined as

$$
\begin{aligned}
& L_{\Psi}:\left(\mathbb{R}^{w_{1}}\right)^{\mathbb{Z}^{2}} \times\left(\mathbb{R}^{w_{2}}\right)^{\mathbb{Z}^{2}} \longrightarrow(\mathbb{R})^{\mathbb{Z}^{2}} \\
& L_{\Psi}(v, w):=\left[\begin{array}{ll}
L_{\Psi_{1}}(v, w) & L_{\Psi_{2}}(v, w)
\end{array}\right]^{\top} .
\end{aligned}
$$

Finally, we introduce the notion of (discrete) divergence of a VBDF. Given a VBDF $L_{\Psi}=\operatorname{col}\left(L_{\Psi_{1}}, L_{\Psi_{2}}\right)^{\top}$, we define its divergence as the BDF defined by

$$
\begin{aligned}
\left(\operatorname{div} L_{\Psi}\right)\left(w_{1}, w_{2}\right) & :=\left(L_{\Psi_{1}}\left(w_{1}, w_{2}\right)-\sigma_{1}\left(L_{\Psi_{1}}\left(w_{1}, w_{2}\right)\right)\right) \\
& +\left(L_{\Psi_{2}}\left(w_{1}, w_{2}\right)-\sigma_{2}\left(L_{\Psi_{2}}\left(w_{1}, w_{2}\right)\right)\right)
\end{aligned}
$$


for all $w_{1}, w_{2}$. It is straightforward to verify that in terms of the 4-variable polynomial matrices associated with the BDF's, the relationship between a VBDF and its divergence is expressed as

$$
\begin{aligned}
\Phi\left(\zeta_{1}, \zeta_{2}, \eta_{1}, \eta_{2}\right)= & \left(1-\zeta_{1} \eta_{1}\right) \Psi_{1}\left(\zeta_{1}, \zeta_{2}, \eta_{1}, \eta_{2}\right) \\
& +\left(1-\zeta_{2} \eta_{2}\right) \Psi_{2}\left(\zeta_{1}, \zeta_{2}, \eta_{1}, \eta_{2}\right)
\end{aligned}
$$

In order to characterize those $\mathrm{BDF}$ s wich are the divergence of some VBDF, we need to introduce the "del" operator, defined as

$$
\begin{aligned}
& \partial: \mathbb{R}^{\mathrm{w}_{1} \times \mathrm{w}_{2}}\left[\zeta_{1}, \zeta_{2}, \eta_{1}, \eta_{2}\right] \longrightarrow \mathbb{R}^{\mathrm{w}_{1} \times \mathrm{w}_{2}}\left[\xi_{1}, \xi_{2}, \xi_{1}^{-1}, \xi_{2}^{-1}\right] \\
& \partial \Phi\left(\xi_{1}, \xi_{2}\right):=\Phi\left(\xi_{1}^{-1}, \xi_{2}^{-1}, \xi_{1}, \xi_{2}\right)
\end{aligned}
$$

The following result holds true.

Proposition 6: A BDF $L_{\Phi}$ is the divergence of some VBDF $L_{\Psi}$ if and only if $\partial \Phi\left(\xi_{1}, \xi_{2}\right)=0$.

The definition and properties described above can be adapted to a vector quadratic difference form (VQDF) in a obvious manner.

We now introduce the notion of positivity of a QDF. We define a QDF $Q_{\Delta}$ induced by a four-variable polynomial matrix $\Delta \in \mathbb{R}^{\mathrm{w} \times \mathrm{w}}\left[\zeta_{1}, \zeta_{2}, \eta_{1}, \eta_{2}\right]$ to be nonnegative if $Q_{\Delta}\left(w\left(x_{1}, x_{2}\right)\right) \geq 0 \forall\left(x_{1}, x_{2}\right) \in \mathbb{Z}^{2}$ and for all $w \in\left(\mathbb{R}^{\mathrm{w}}\right)^{\mathbb{Z}^{2}}$. This will be denoted with $Q_{\Delta} \geq 0$ or $\Delta(\zeta, \eta) \geq 0$. We call $Q_{\Delta}$ positive, denoted $Q_{\Delta}>0$ or $\Delta(\zeta, \eta)>0$, if $Q_{\Delta} \geq 0$ and $Q_{\Delta}\left(w\left(x_{1}, x_{2}\right)\right)=0 \forall\left(x_{1}, x_{2}\right)$ implies $w=0$. Often in the following we will also consider QDFs induced by matrices of the form $\Delta\left(e^{-i \omega}, \zeta_{2}, e^{i \omega}, \eta_{2}\right)$, i.e. matrices in the indeterminates $\zeta_{2}, \eta_{2}$ with coefficients being polynomials in $e^{i \omega}$ for some $\omega \in \mathbb{R}$. The definition of nonnegativity and positivity in this case is readily adapted from the above definition.

Finally, we define equivalence of QDFs along a behavior. Let $\mathfrak{B} \in \mathcal{L}_{2}^{\mathrm{W}}$ and $\Phi_{i} \in \mathbb{R}^{\mathrm{w} \times \mathrm{w}}\left[\zeta_{1}, \zeta_{2}, \eta_{1}, \eta_{2}\right], i=1,2$. Then $Q_{\Phi_{1}}$ is equivalent modulo $\mathfrak{B}$ to $Q_{\Phi_{2}}$, denoted $Q_{\Phi_{1}} \stackrel{\mathfrak{B}}{=} Q_{\Phi_{2}}$, if $Q_{\Phi_{1}}(w)=Q_{\Phi_{2}}(w)$ for all $w \in \mathfrak{B}$. Now let $\mathfrak{B}=$ ker $R\left(\sigma_{1}, \sigma_{2}\right)$; then it can be shown that $Q_{\Phi_{1}} \stackrel{\mathfrak{B}}{=} Q_{\Phi_{2}}$ if and only if there exists $X \in \mathbb{R}^{\bullet \times \mathrm{w}}\left[\zeta_{1}, \zeta_{2}, \eta_{1}, \eta_{2}\right]$ such that

$$
\begin{aligned}
\Phi_{1}\left(\zeta_{1}, \zeta_{2}, \eta_{1}, \eta_{2}\right)= & \Phi_{2}\left(\zeta_{1}, \zeta_{2}, \eta_{1}, \eta_{2}\right) \\
& +R^{\top}\left(\zeta_{1}, \zeta_{2}\right) X\left(\zeta_{1}, \zeta_{2}, \eta_{1}, \eta_{2}\right) \\
& +X^{\top}\left(\eta_{1}, \eta_{2}, \zeta_{1}, \zeta_{2}\right) R\left(\eta_{1}, \eta_{2}\right)
\end{aligned}
$$

(see Proposition 10 in [8]). In this case we also write $\Phi_{1}\left(\zeta_{1}, \zeta_{2}, \eta_{1}, \eta_{2}\right)=\Phi_{2}\left(\zeta_{1}, \zeta_{2}, \eta_{1}, \eta_{2}\right) \bmod R$, or $\Phi_{1}\left(\zeta_{1}, \zeta_{2}, \eta_{1}, \eta_{2}\right)-\Phi_{2}\left(\zeta_{1}, \zeta_{2}, \eta_{1}, \eta_{2}\right)=0 \bmod R$.

\section{NECESSARY AND SUFFICIENT CONDITIONS FOR STABILITY OF 2-D SYSTEMS}

We establish the main result of this paper, a Lyapunov necessary and sufficient condition for an autonomous square behavior $\mathfrak{B} \in \mathcal{L}_{2}^{\mathrm{W}}$ to be asymptotically stable. Using the result of Theorem 5 , which allows us to bring $\mathcal{K}$-stability for a general proper cone $\mathcal{K}$ back to stability on the first orthant, in this section we concentrate on stability with respect to the proper cone consisting of the first orthant of $\mathbb{Z} \times \mathbb{Z}$. We will denote this set with $\mathcal{K}_{0}$ in the following. Moreover, for the time being we concentrate on the case of square autonomous systems.

We begin this section with a straightforward but important refinement of Proposition 3.5 of [17].

Proposition 7: Let $\mathfrak{B} \in \mathcal{L}_{2}^{\mathrm{W}}$ be square and autonomous, and let $\mathfrak{B}=\operatorname{ker} \Delta\left(\sigma_{1}, \sigma_{2}\right)$ with $\Delta \in \mathbb{R}^{\mathrm{w} \times \mathrm{w}}\left[\xi_{1}, \xi_{2}\right]$ nonsingular. Assume that $\delta:=\operatorname{det} \Delta$ is such that $\mathcal{H}_{\delta}$ is a subset of $\mathcal{K}_{0}$, the first orthant of $\mathbb{Z} \times \mathbb{Z}$, that intersects the coordinate axes only in the origin. Then the following statements are equivalent:

1) $\mathfrak{B}$ is $\mathcal{K}_{0}$-stable;

2) For all $\omega \in \mathbb{R}$, the polynomial $\delta\left(e^{j \omega}, \xi_{2}\right)$ has all its roots outside of the closed unit disk $\left\{z_{2} \in \mathbb{C}|| z_{2} \mid \geq\right.$ $1\}$, and the polynomial $\delta\left(\xi_{1}, e^{j \omega}\right)$ has all its roots outside of the closed unit disk $\left\{z_{1} \in \mathbb{C}|| z_{1} \mid \geq 1\right\}$.

Proof: The proof follows from Theorem 5 and from the equivalence of statements $i$ ) and $i v$ ) in Proposition 3.1 of [6].

We now state a condition equivalent to that of Proposition 7 in terms of a pair of quadratic difference forms satisfying a Lyapunov-type equation. In order to do this, we introduce first some notation; in the following we denote with $\mathrm{Per}_{2} \subset$ $\left(\mathbb{R}^{\mathrm{w}}\right)^{\mathbb{Z}^{2}}$ the set consisting of all trajectories $v \in\left(\mathbb{R}^{\mathrm{w}}\right)^{\mathbb{Z}^{2}}$ such that the restriction of $v$ to the lines $\left\{\left(x_{1}, x_{2}\right) \mid x_{2} \in \mathbb{Z}\right\}$ is periodic for all fixed $x_{1} \in \mathbb{Z}$, i.e.

$$
\begin{aligned}
\operatorname{Per}_{2}:= & \left\{v \in\left(\mathbb{R}^{\mathrm{w}}\right)^{\mathbb{Z}^{2}} \mid v\left(x_{1}, \cdot\right) \in\left(\mathbb{R}^{\mathrm{w}}\right)^{\mathbb{R}}\right. \text { is periodic } \\
& \text { for all fixed } \left.x_{1} \in \mathbb{Z}\right\}
\end{aligned}
$$

and analogously we define

$$
\begin{aligned}
\operatorname{Per}_{1}:= & \left\{v \in\left(\mathbb{R}^{\mathrm{w}}\right)^{\mathbb{Z}^{2}} \mid v\left(\cdot, x_{2}\right) \in\left(\mathbb{R}^{\mathrm{w}}\right)^{\mathbb{R}}\right. \text { is periodic } \\
& \text { for all fixed } \left.x_{2} \in \mathbb{Z}\right\}
\end{aligned}
$$

The main result of this section is the following.

Theorem 8: Let $\mathfrak{B}$ be a 2-D square autonomous linear differential behavior, i.e. $\mathfrak{B}=\operatorname{ker} R\left(\sigma_{1}, \sigma_{2}\right)$. Then the following statements are equivalent:

1) $\mathfrak{B}$ is $\mathcal{K}_{0}$-stable.

2) There exists a VQDF $Q_{\Phi}=\operatorname{col}\left(Q_{\Phi_{1}}, Q_{\Phi_{2}}\right)$ and a QDF $Q_{\Delta}$ such that

(2a) $\operatorname{div} Q_{\Phi} \stackrel{\mathfrak{B}}{=}-Q_{\Delta}$;

(2b) $Q_{\Phi_{1}}(w), Q_{\Delta}(w)>0$ for all $w \in \mathfrak{B} \cap \operatorname{Per}_{2}$, and $Q_{\Phi_{2}}(w), Q_{\Delta}(w)>0$ for all $w \in \mathfrak{B} \cap$ Per $_{1}$.

3) There exist $\Phi=\operatorname{col}\left(\Phi_{1}, \Phi_{2}\right)$ and $\Delta$, with $\Phi_{1}, \Phi_{2}, Y \in$ $\mathbb{R}^{\mathrm{w} \times \mathrm{w}}\left[\zeta_{1}, \zeta_{2}, \eta_{1}, \eta_{2}\right], \Delta \in \mathbb{R}_{s}^{\mathrm{w} \times \mathrm{w}}\left[\zeta_{1}, \zeta_{2}, \eta_{1}, \eta_{2}\right]$ such that

(3a) $\quad\left(1-\zeta_{1} \eta_{1}\right) \Phi_{1}\left(\zeta_{1}, \zeta_{2}, \eta_{1}, \eta_{2}\right)$

$+\left(1-\zeta_{2} \eta_{2}\right) \Phi_{2}\left(\zeta_{1}, \zeta_{2}, \eta_{1}, \eta_{2}\right)$ $=-\Delta\left(\zeta_{1}, \zeta_{2}, \eta_{1}, \eta_{2}\right)$

$+R\left(\zeta_{1}, \zeta_{2}\right)^{\top} Y\left(\zeta_{1}, \zeta_{2}, \eta_{1}, \eta_{2}\right)$

$+Y\left(\eta_{1}, \eta_{2}, \zeta_{1}, \zeta_{2}\right)^{\top} R\left(\eta_{1}, \eta_{2}\right)$;

$(3 b)$

$$
\begin{aligned}
& \Phi_{1}\left(\zeta_{1}, \zeta_{2}, \eta_{1}, \eta_{2}\right) \stackrel{\text { B } P \text { Per } 2}{>} 0 \text {, } \\
& \Phi_{2}\left(\zeta_{1}, \zeta_{2}, \eta_{1}, \eta_{2}\right) \stackrel{\mathfrak{B} \cap \operatorname{Per}_{1}}{>} 0, \\
& \Delta\left(\zeta_{1}, \zeta_{2}, \eta_{1}, \eta_{2}\right) \stackrel{\mathcal{B}^{\prime} \operatorname{Per}_{i}}{>} 0, i=1,2 .
\end{aligned}
$$


We refer to a VQDF $Q_{\Phi}=\operatorname{col}\left(Q_{\Phi_{1}}, Q_{\Phi_{2}}\right)$ satisfying (2a) and $(2 \mathrm{~b})$ as a Lyapunov function for $\mathfrak{B}$.

Proof: The equivalence of statements (2) and (3) is straightforward.

We now prove the implication $(3) \Longrightarrow(1)$. Consider any trajectory in $\mathfrak{B}$ of the form $w\left(t_{1}, t_{2}\right)=v \lambda^{t_{1}} \mu^{t_{2}}$ for some $v \in \mathbb{C}^{\mathrm{w}}$ and $\lambda, \mu \in \mathbb{C}$. We now prove that if $\mu$ lies on the unit circle, i.e. $\mu=e^{i \omega}$ for some $\omega \in \mathbb{R}$, then $|\lambda|>1$. Once this will have been established, statement (1) follows from Proposition 7. $(3 a)$ :

Let $\zeta_{1}=\bar{\lambda}, \eta_{1}=\lambda, \zeta_{2}=\bar{\mu}=e^{-i \omega}, \eta_{2}=\mu=e^{i \omega}$ in $(1-\bar{\lambda} \lambda) v^{\top} \Phi_{1}\left(\bar{\lambda}, e^{-i \omega}, \lambda, e^{i \omega}\right) v=-v^{\top} \Delta\left(\bar{\lambda}, e^{-i \omega}, \lambda, e^{i \omega}\right) v$

The right-hand side of this equation is strictly negative; on the left-hand side $v^{\top} \Phi_{1}\left(\bar{\lambda}, e^{-i \omega}, \lambda, e^{i \omega}\right) v>0$, and consequently it follows that $1-\bar{\lambda} \lambda<0$. An analogous argument is used when $w\left(t_{1}, t_{2}\right)=v e^{i \omega t_{1}} \mu^{t_{2}}$. This proves the claim.

The proof of implication (1) $\Longrightarrow(3)$ is established by producing matrices $\Phi_{i} \in \mathbb{R}_{S}^{\mathrm{w} \times \mathrm{w}}\left[\zeta_{1}, \zeta_{2}, \eta_{1}, \eta_{2}\right], i=1,2$, and $\Delta \in \mathbb{R}_{S}^{\mathrm{W} \times \mathrm{w}}\left[\zeta_{1}, \zeta_{2}, \eta_{1}, \eta_{2}\right]$ such that $(3 a)-(3 b)$ hold.

Write $R\left(\xi_{1}, \xi_{2}\right)=\sum_{i=0}^{L_{1}} R_{i}\left(\xi_{2}\right) \xi_{1}^{L_{1}}=\sum_{i=0}^{L_{2}} R_{i}^{\prime}\left(\xi_{1}\right) \xi_{2}^{L_{2}}$, where $L_{i}$ is the highest power of $\xi_{i}$ in $R, i=1,2$. Define the four-variable polynomial matrix

$$
\begin{aligned}
H\left(\zeta_{1}, \zeta_{2}, \eta_{1}, \eta_{2}\right) & :=R\left(\zeta_{1}, \zeta_{2}\right)^{\top} R\left(\eta_{1}, \eta_{2}\right) \\
& -\zeta_{1}^{L_{1}} \zeta_{2}^{L_{2}} \eta_{1}^{L_{1}} \eta_{2}^{L_{2}} R\left(\eta_{1}^{-1}, \eta_{2}^{-1}\right)^{\top} R\left(\zeta_{1}^{-1}, \zeta_{2}^{-1}\right) .
\end{aligned}
$$

Observe that $\partial H=0$; conclude from Proposition 6 that there exists $\Phi=\operatorname{col}\left(\Phi_{1}, \Phi_{2}\right) \in \mathbb{R}^{2 \mathrm{w} \times \mathrm{w}}\left[\zeta_{1}, \zeta_{2}, \eta_{1}, \eta_{2}\right]$ such that $\left(1-\zeta_{1} \eta_{1}\right) \Phi_{1}\left(\zeta_{1}, \zeta_{2}, \eta_{1}, \eta_{2}\right)+\left(1-\zeta_{2} \eta_{2}\right) \Phi_{2}\left(\zeta_{1}, \zeta_{2}, \eta_{1}, \eta_{2}\right)=$ $H\left(\zeta_{1}, \zeta_{2}, \eta_{1}, \eta_{2}\right)$. Moreover, it is easy to see using Proposition 3.2 of [7] that

$$
\begin{aligned}
& \Phi_{1}\left(\zeta_{1}, e^{-i \omega}, \eta_{1}, e^{i \omega}\right)= \\
& \frac{R\left(\zeta_{1}, e^{-i \omega}\right)^{\top} R\left(\eta_{1}, e^{i \omega}\right)-\zeta_{1}^{L_{1}} \eta_{1}^{L_{1}} R\left(\eta_{1}^{-1}, e^{-i \omega}\right)^{\top} R\left(\zeta_{1}^{-1}, e^{i \omega}\right)}{1-\zeta_{1} \eta_{1}} .
\end{aligned}
$$

From Proposition 7 it follows that since $\mathfrak{B}$ is $\mathcal{K}_{0}$-stable the polynomial $\operatorname{det}\left(R\left(\xi_{1}, e^{i \omega}\right)\right)$ is anti-Schur (meaning all its roots have modulus greater than one) for all $\omega \in \mathbb{R}$. It follows from Corollary 1 of [9] that for all $\omega \in \mathbb{R}$ $\Phi_{1}\left(\zeta_{1}, e^{-i \omega}, \eta_{1}, e^{i \omega}\right)>0$, since (4) is equivalent with $\Phi_{1}$ being the $R$-canonical solution of an $\omega$-dependent polynomial Lyapunov equation in two variables (see equation (4) of [9]) for the behavior described in kernel form by $R\left(\xi_{1}, e^{i \omega}\right)$. From this it follows that $\Phi_{1} \stackrel{\mathfrak{B} \cap \mathrm{Per}_{2}}{>} 0$.

An analogous argument based on the same considerations and on the fact that $R\left(e^{i \omega}, \xi_{2}\right)$ is anti-Schur for all $\omega \in \mathbb{R}$, shows that $\Phi_{2}\left(e^{-i \omega}, \zeta_{2}, e^{i \omega}, \eta_{2}\right)>0$ for all $\omega \in \mathbb{R}$.

In order to conclude the proof, define

$$
Y\left(\xi_{1}, \xi_{2}\right):=\frac{1}{2} R\left(\xi_{1}, \xi_{2}\right)
$$

$\Delta\left(\zeta_{1}, \zeta_{2}, \eta_{1}, \eta_{2}\right):=\zeta_{1}^{L_{1}} \eta_{1}^{L_{1}} \zeta_{2}^{L_{2}} \eta_{2}^{L_{2}} R\left(\eta_{1}^{-1}, \eta_{2}^{-1}\right)^{\top} R\left(\zeta_{1}^{-1}, \zeta_{2}^{-1}\right)$
The fact that $\Delta\left(\zeta_{1}, e^{-i \omega}, \eta_{1}, e^{i \omega}\right)>0$ and $\Delta\left(e^{-i \omega}, \zeta_{2}, e^{i \omega}\right.$, $\left.\eta_{2}\right)>0$ for all $\omega \in \mathbb{R}$ follows from the $\mathcal{K}_{0}$-stability of $\mathfrak{B}$, which implies for all $\omega \in \mathbb{R}$ that $R\left(\xi_{1}, e^{i \omega}\right)$ and $R\left(e^{i \omega}, \xi_{2}\right)$ are anti-Schur.

The 4-variable polynomial matrices $\Phi=\operatorname{col}\left(\Phi_{1}, \Phi_{2}\right)$ and $\Delta$ given in the proof of Theorem 8 can be considered as an $\omega$ parametrized 2-D discrete-time version of the multivariable Bézoutian

$$
\frac{R(\zeta)^{\top} R(\eta)-R(-\eta)^{\top} R(-\zeta)}{\zeta+\eta}
$$

used in analyzing stability of 1-D continuous-time systems. In the single-variable (i.e. $\mathrm{w}=1$ ) case, stability conditions based on the positivity of the coefficient matrix of an $\omega$ dependent Bézoutian have been obtained in [5], [6]. Of course, there are more Lyapunov functions than only the Bézoutian, as the following example shows.

Example 9: Consider the system described in kernel form by the polynomial

$$
p\left(\zeta_{1}, \zeta_{2}, \eta_{1}, \eta_{2}\right):=1+\frac{1}{2} \xi_{1}+\frac{1}{2} \xi_{2}+\frac{1}{2} \xi_{1} \xi_{2}
$$

It can be verified that the Bézoutian equals

$$
\begin{aligned}
B\left(\zeta_{1}, \zeta_{2}, \eta_{1}, \eta_{2}\right) & =\left(1-\zeta_{1} \eta_{1}\right) \underbrace{\frac{1}{2}\left(\eta_{2}+\zeta_{2}+3 \eta_{2} \zeta_{2}\right)}_{=: \Psi_{1}\left(\zeta_{1}, \zeta_{2}, \eta_{1}, \eta_{2}\right)} \\
& +\left(1-\zeta_{2} \eta_{2}\right) \underbrace{\frac{1}{4}\left(3+\eta_{1}+\zeta_{1}\right)}_{=: \Psi_{2}\left(\zeta_{1}, \zeta_{2}, \eta_{1}, \eta_{2}\right)}
\end{aligned}
$$

It is easy to see that $\Psi_{1}\left(\zeta_{1}, e^{-i \omega}, \eta_{1}, e^{i \omega}\right)=\frac{1}{4}(3+$ $2 \cos (\omega))=\Psi_{2}\left(e^{-i \omega}, \zeta_{2}, e^{i \omega}, \eta_{2}\right)>0$ for all $\omega \in \mathbb{R}$, and consequently the system is stable.

We now give another Lyapunov functional for ker $p\left(\sigma_{1}, \sigma_{2}\right)$; define the two-variable polynomial

$$
\Delta^{\prime}\left(\zeta_{1}, \zeta_{2}, \eta_{1}, \eta_{2}\right):=-\frac{3}{2}-\frac{1}{4}\left(\zeta_{1}+\eta_{1}+\zeta_{2}+\eta_{2}\right)
$$

and observe that $\Delta^{\prime}\left(\zeta_{1}, e^{-i \omega}, \eta_{1}, e^{i \omega}\right)<0$ for all $\omega \in \mathbb{R}$, and that an analogous inequality holds for $\Delta^{\prime}\left(e^{-i \omega}, \zeta_{2}, e^{i \omega}, \eta_{2}\right)$. Define also

$$
\begin{aligned}
\Psi_{1}^{\prime}\left(\zeta_{1}, \zeta_{2}, \eta_{1}, \eta_{2}\right) & :=\frac{1}{4}\left(1+\zeta_{2}\right)\left(1+\eta_{2}\right) \\
\Psi_{2}^{\prime}\left(\zeta_{1}, \zeta_{2}, \eta_{1}, \eta_{2}\right) & :=\frac{1}{4}\left(1+\zeta_{1}\right)\left(1+\eta_{1}\right)
\end{aligned}
$$

and observe that $\Psi_{1}^{\prime}\left(\zeta_{1}, e^{-i \omega}, \eta_{1}, e^{i \omega}\right)=\frac{1}{4}\left|1+e^{i \omega}\right|^{2}=$ $\Psi_{2}^{\prime}\left(e^{-i \omega}, \zeta_{2}, e^{i \omega}, \eta_{2}\right)>0$ for all $\omega \in \mathbb{R}$. It is easy to verify that

$$
\frac{1}{4}\left[\begin{array}{l}
\left(1+\zeta_{2}\right)\left(1+\eta_{2}\right) \\
\left(1+\zeta_{1}\right)\left(1+\eta_{1}\right)
\end{array}\right]
$$

is a Lyapunov function for $\mathfrak{B}=\operatorname{ker} p\left(\sigma_{1}, \sigma_{2}\right)$ with divergence equal to $-\frac{3}{2}-\frac{1}{4}\left(\zeta_{1}+\eta_{1}+\zeta_{2}+\eta_{2}\right)$ along $\mathfrak{B}$.

We conclude this section with a discussion on the 2-D polynomial Lyapunov equation, which we now introduce. 
Consider the " $\nabla$ " map associating a pair of 4-variable polynomial matrices $\left(\Psi_{1}\left(\zeta_{1}, \zeta_{2}, \eta_{1}, \eta_{2}\right), \Psi_{2}\left(\zeta_{1}, \zeta_{2}, \eta_{1}, \eta_{2}\right)\right)$ to the polynomial matrix

$$
\begin{aligned}
\nabla\left[\begin{array}{l}
\Psi_{1}\left(\zeta_{1}, \zeta_{2}, \eta_{1}, \eta_{2}\right) \\
\Psi_{2}\left(\zeta_{1}, \zeta_{2}, \eta_{1}, \eta_{2}\right)
\end{array}\right]:= & \left(1-\zeta_{1} \eta_{1}\right) \Psi_{1}\left(\zeta_{1}, \zeta_{2}, \eta_{1}, \eta_{2}\right) \\
& +\left(1-\zeta_{2} \eta_{2}\right) \Psi_{2}\left(\zeta_{1}, \zeta_{2}, \eta_{1}, \eta_{2}\right)
\end{aligned}
$$

It is easy to see that $\nabla$ is linear; moreover, observe that condition $(3 a)$ of Theorem 8 can be rewritten using $\nabla$ as

$$
\nabla\left[\begin{array}{l}
\Phi_{1}\left(\zeta_{1}, \zeta_{2}, \eta_{1}, \eta_{2}\right) \\
\Phi_{2}\left(\zeta_{1}, \zeta_{2}, \eta_{1}, \eta_{2}\right)
\end{array}\right]=-\Delta\left(\zeta_{1}, \zeta_{2}, \eta_{1}, \eta_{2}\right) \bmod R .
$$

We call (5) the 2-D polynomial Lyapunov equation (2-D PLE in the following), by analogy with the equation studied in the $1-D$ case in section 4 of [18] and in [12]. It follows from this discussion that a fourth condition equivalent with those stated in Theorem 8 can be given for the $\mathcal{K}_{0}$-stability of a square autonomous behavior $\mathfrak{B}$, namely:

4. There exist $\Phi=\operatorname{col}\left(\Phi_{1}, \Phi_{2}\right)$ and $\Delta$, with $\Phi_{1}, \Phi_{2} \in$ $\mathbb{R}^{\mathrm{w} \times \mathrm{w}}\left[\zeta_{1}, \zeta_{2}, \eta_{1}, \eta_{2}\right], \Delta \in \mathbb{R}_{s}^{\mathrm{w} \times \mathrm{w}}\left[\zeta_{1}, \zeta_{2}, \eta_{1}, \eta_{2}\right]$ such that

(4a) The 2-D PLE (5) is satisfied;

$$
\begin{aligned}
& \Phi_{1}\left(\zeta_{1}, \zeta_{2}, \eta_{1}, \eta_{2}\right) \stackrel{\mathfrak{B} \cap \mathrm{Per}_{2}}{>} 0 \text {, } \\
& \Phi_{2}\left(\zeta_{1}, \zeta_{2}, \eta_{1}, \eta_{2}\right) \stackrel{\mathfrak{B} \cap \mathrm{Per}_{1}}{>} 0, \\
& \Delta\left(\zeta_{1}, \zeta_{2}, \eta_{1}, \eta_{2}\right) \stackrel{\mathfrak{B}^{\circ} \mathrm{Per}_{i}}{>} 0, i=1,2 .
\end{aligned}
$$

\section{CONCLUSIONS}

The main result of this paper is Theorem 8, which states necessary and sufficient conditions for the asymptotic stability of a 2-D behavior in the sense of Valcher. Two topics are currently being investigated: to determine conditions for the solvability of the 2-D-PLE in the multivariable case; and to develop algorithms for the efficient solution of the equation.

\section{ACKNOWLEDGMENTS}

The second author gratefully acknowledges the financial support of The Great Britain Sasakawa Foundation for financially supporting the visit to Kyoto University during which the results presented in this paper were obtained. The first author also acknowledges that this work is partly supported by Grant-in-Aid for Young Scientists (Start-up) No. 20860025 of Japan Society for the Promotion of Science.

\section{REFERENCES}

[1] E. Fornasini and G. Marchesini, "Stability Analysis of 2-D Systems", IEEE Trans. Circ. Syst., vol. CAS-27. no. 12, pp. 1210-1217, 1980.

[2] E. Fornasini, P. Rocha, and S. Zampieri, "State-space realization of 2-D finite-dimensional behaviors", SIAM J. Contr. Opt., vol. 31, pp. 1502-1517, 1993.

[3] E. Fornasini, M.E. Valcher, " $n D$ polynomial matrices with applications to multidimensional signal analysis", Multidimensional Syst. Sign. Proc., vol. 8, pp. 387-407, 1997.

[4] P.A. Fuhrmann, A polynomial approach to linear algebra, SpringerVerlag, NY, 1996.

[5] J.S. Geronimo and H.J. Woerdeman, "Positive extensions, Fejér-Riesz factorization and autoregressive filters in two-variables", Ann. Math., vol. 160, pp. 839-906, 2004.

[6] J.S. Geronimo and H.J. Woerdeman, "Two-Variable Polynomials: Intersecting Zeros and Stability", IEEE Trans. Circ. Syst.- I: Regular Papers, vol. 53, no. 5, pp. 1130-1139,2006.
[7] O. Kaneko and T. Fujii, "Discrete-time average positivity and spectral factorization in a behavioral framework", Systems and control letters, vol. 39, pp. 31-44, 2000.

[8] C. Kojima, P. Rapisarda, and K. Takaba, "Canonical forms for polynomial and quadratic differential operators," Systems and Control Letters, vol. 56, pp. 678-684, 2007.

[9] C. Kojima and K. Takaba, "A Generalized Lyapunov Stability Theorem for Discrete-time Systems based on Quadratic Difference Forms", in Proc. 44th IEEE Conference on Decision and Control, and the European Control Conference, pp. 2911-2916, Seville, Spain, 2005.

[10] C. Kojima and K. Takaba, "A Lyapunov Stability Analysis of 2D Discrete-Time Behaviors", in Proc. 17-th MTNS, pp. 2504-2512, Kyoto, Japan, 2006.

[11] W.-S. Lu, E. B. Lee, "Stability Analysis of Two-Dimensional Systems via a Lyapunov Approach", IEEE Trans. Circ. Syst., vol. CAS-32, no. 1, pp. 61-68, 1985

[12] R. Peeters and P. Rapisarda, "A two-variable approach to solve the polynomial Lyapunov equation", System and Control Letters, 42, pp. 117-126, 2001.

[13] H.K. Pillai and S. Shankar, "A behavioral approach to control of distributed systems", SIAM J. Control Opt., vol. 37, no. 2, pp. 388-408, 1999.

[14] H. K. Pillai and J. C. Willems, "Lossless and dissipative distributed systems”, SIAM J. Control Optim., vol. 40, pp. 1406-1430, 2002.

[15] J.W. Polderman and J.C. Willems, Introduction to mathematical system theory: a behavioral approach, Springer-Verlag, Berlin, 1997.

[16] P. Rocha, Structure and representation of 2-D systems, Ph.D. thesis, Univ. of Groningen, The Netherlands, 1990.

[17] M.E. Valcher, "Characteristic Cones and Stability Properties of TwoDimensional Autonomous Behaviors", IEEE Trans. Circ. Syst.-Part I: Fundamental Theory and Applications, vol. 47, no. 3, pp. 290-302, 2000.

[18] J.C. Willems and H.L. Trentelman, "On quadratic differential forms", SIAM J. Control Opt., vol. 36, no. 5, pp. 1703-1749, 1998.

[19] E. Zerz, "Multidimensional Behaviors: An Algebraic Approach to Control Theory for PDE", International Journal of Control, vol. 77, pp. 812-820, 2004. 